\title{
Current status of robot-assisted thoracoscopic surgery in Japan
}

\author{
Tomohiro Haruki, Yuzo Takagi, Yasuaki Kubouchi, Yoshiteru Kidokoro, Atsuyuki Nakanishi, \\ Yuji Taniguchi, Hiroshige Nakamura
}

Division of General Thoracic Surgery, Department of Surgery, Faculty of Medicine, Tottori University, Tottori, Japan

Contributions: (I) Conception and design: T Haruki; (II) Administrative support: All authors; (III) Provision of study materials or patients: T Haruki;

(IV) Collection and assembly of data: All authors; (V) Data analysis and interpretation: All authors; (VI) Manuscript writing: All authors; (VII) Final approval of manuscript: All authors.

Correspondence to: Tomohiro Haruki, MD, PhD. 36-1, Nishi-cho, Yonago, Tottori 683-8504, Japan. Email: tomohiroh@tottori-u.ac.jp.

\begin{abstract}
Since approximately 2000, when surgical robotic systems were first introduced, robot-assisted thoracoscopic surgery (RATS) has been adopted by dozens of major institutes, particularly in the United States and several European countries. In Japan, video-assisted thoracoscopic surgery (VATS), especially multiport VATS, was developed and standardized in various facilities during this period. In April 2018, RATS lobectomy and resection of mediastinal tumors were started to be covered by national health insurance. In Japan, the number of RATS procedures has increased rapidly in recent days, and it is expected to become more common and familiar to Japanese thoracic surgeons of all generations. The da Vinci Surgical System has several well-known clear technical advantages, such as a three-dimensional visual field that can be magnified up to 10 times, articulated joint forceps with seven degrees of freedom, and a motionscaling function that prevents camera and instrument shaking. These advantages provide thoracic surgeons with higher-quality instrument maneuverability and general comfort during surgery. While there are many technical advantages in RATS, there are also many non-technical skills that thoracic surgeons should be aware of, such as risk management of intraoperative bleeding. In this review article, we outline the current status of RATS for lung cancer and mediastinal tumor excision in Japan and discuss various issues and the future perspectives of robotic surgery.
\end{abstract}

Keywords: Robot-assisted thoracoscopic surgery (RATS); lung cancer; mediastinal tumor

Received: 25 November 2019; Accepted: 24 December 2019; Published: 05 July 2020.

doi: $10.21037 /$ jovs.2019.12.08

View this article at: http://dx.doi.org/10.21037/jovs.2019.12.08

\section{Introduction}

Since approximately 2000, when surgical robotic systems were first introduced, robot-assisted thoracoscopic surgery (RATS) has been adopted by dozens of major institutes, particularly in the United States of America (USA) and several European countries. The da Vinci Surgical System (DVSS; Intuitive Surgical Company, Sunnyvale, USA), which is the only robotic surgical apparatus marketed for RATS, has several well-known clear technical advantages, such as a three-dimensional visual field that can be magnified up to 10 times, articulated joint forceps with seven degrees of freedom, and a motion-scaling function that prevents camera and instrument shaking. These advantages of robotic surgical systems provide thoracic surgeons with higher-quality instrument maneuverability and general comfort during surgery. In thoracic surgery, primary lung cancer and various mediastinal neoplastic diseases are good operative indications for RATS. The number of robotic surgical procedures has been increasing, and by the end of 2015, the percentage of RATS lobectomies in the USA was approximately $15 \%$, with over 400 surgeons performing RATS.

In Japan, video-assisted thoracoscopic surgery (VATS), rather than RATS, has evolved since 2000, with technical innovations in endoscopic instruments and scopes, as well as the standardization of surgical procedures. RATS was actually first performed as a "non-health insurance coverage 
treatment" at limited institutions in Japan, beginning in 2011, later than in Western countries. In April 2018, 7 years after its initial introduction in Japan, RATS lobectomy and resection of mediastinal tumors were covered by the national health insurance system. The number of RATS procedures has since increased rapidly, and we expect that more than 2,000 patients will undergo RATS over the next year. Considering the current situation, RATS is at a turning point in Japan.

In this review article, we outline the current status of robotic surgery for lung cancer and mediastinal tumor excision in Japan and discuss various issues and the future prospects of robotic surgery.

\section{Review}

\section{The history of RATS in Japan}

A small number of reports on thoracic robotic surgery in Japan were published in 2000 when RATS was launched and beginning to spread in the USA and European countries. As initial experiences, Yoshino et al. reported RATS resection of anterior and posterior mediastinal tumors at the beginning of the 2000s $(1,2)$; however, no notable reports of RATS from Japan have been published since then. The Japanese public medical insurance system involves complicated application procedures for new surgical apparatuses to be used in clinical practice. Because unapproved materials and procedures are not covered by the national health insurance, it is difficult for surgeons to use novel surgical technologies; therefore, RATS did not evolve considerably in Japan during the first decade of the 2000s. Instead, VATS, especially multiport VATS, was developed and standardized in various facilities across Japan during this period. The high-definition VATS endoscope provides clearer microstructural images, the endostapler enables precise large vessel and lung parenchyma processing, and the energy devices make it easier to seal small vessels, cut tissue, and stop bleeding. Skilled Japanese thoracic surgeons made full use of these technologies to perform various advanced surgeries using VATS, reported excellent outcomes that are equivalent to thoracotomy regarding surgical and oncological considerations, and established a dominant position of VATS as minimally invasive surgery for lung cancer and other thoracic diseases (3-8). Under these circumstances, the da Vinci S surgical robot was approved as a surgical apparatus with domestic production and a distribution license, by the Pharmaceutical
Affairs Council of the Ministry of Health, Labour and Welfare in Japan, in November 2009. The first patient undergoing RATS lobectomy and mediastinal lymph node dissection for primary lung cancer was reported by Suda et al. in 2010 (9). After approval, DVSS was purchased and introduced by some research and educational institutions such as university hospitals in Japan; however, RATS for chest diseases was still a "non-health insurance coverage procedure", and continued as a patient-paid or hospitalpaid treatment only at the abovementioned institutions. Nakamura et al. summarized the initial 112 cases of RATS performed during this period in Japan and reported that RATS was introduced safely, with a low incidence of postoperative complications and no operation-related mortality (10). In addition to a report showing an equivalent low rate of postoperative complications compared with VATS, the Japanese Association for Chest Surgery also arranged several supports for RATS, such as the creation of guidelines, and establishing proctors and a case registry. Thanks to the efforts of several Japanese thoracic surgeons and others, RATS lobectomy for lung malignant tumors and resection of mediastinal tumors was approved as an insurable procedure by the national health insurance system in April 2018. Since approval, robotic surgery has spread rapidly in Japan and is now performed by many thoracic surgeons. As of April 2018, there were approximately 300 robots and 150 licensees in Japan, and more than 100 RATS cases per month were performed in more than 40 facilities. These numbers will increase, and RATS is expected to become more common and familiar to Japanese thoracic surgeons of all generations.

\section{Setup and techniques in RATS}

Regarding the setup and techniques for RATS for lung cancer, Veronesi et al. introduced different setups and techniques for robotic lobectomy in a review article. The authors reported three major styles: the Park/Veronesi style (11), Cerfolio style (12), and Dylewski style (13) regarding port placement and techniques. These styles have different setups regarding the numbers and locations of the ports, with/without complete portal surgery, and with/without carbon dioxide $\left(\mathrm{CO}_{2}\right)$ insufflation. Some Japanese surgeons also introduced their own setups and techniques. Suda et al. reported using three ports and a $3-\mathrm{cm}$ incision with the GelPOINT Mini (Applied Medical, Rancho Santa Margarita, USA), and performed RATS with $\mathrm{CO}_{2}$ insufflation (14). Taniguchi et al. reported that their setup and techs were 

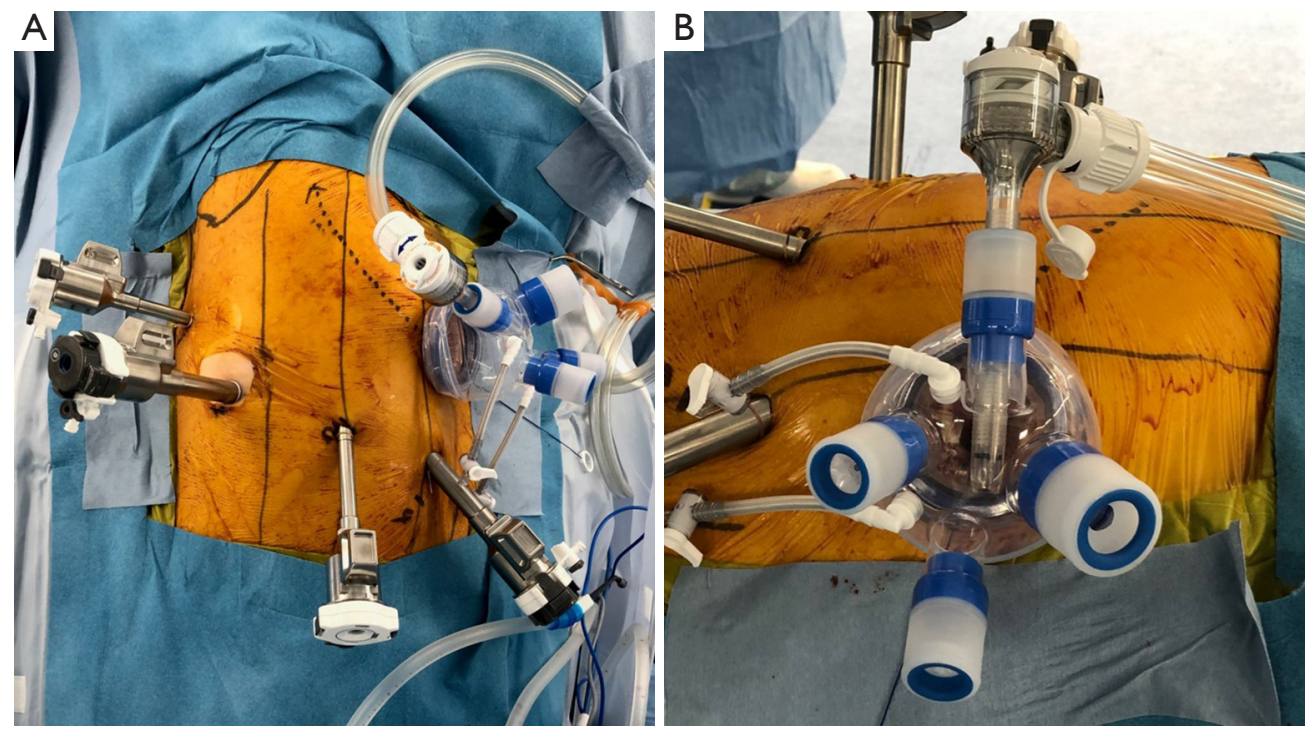

Figure 1 Setups in RATS. (A) Linear ports placed in the eighth intercostal space and the assist port placed in the fifth intercostal space on the anterior axillary line; (B) alnote-LAPSINGLE (Alfresa; Osaka, Japan) and AirSeal (ConMed, Utica, USA) access ports in the assist window. RATS, robot-assisted thoracoscopic surgery.

transitioned from a three-arm to a four-arm technique and from without $\mathrm{CO}_{2}$ insufflation to completely portal surgery with $\mathrm{CO}_{2}$ insufflation (15). Kanzaki et al. reported performing RATS lobectomy with four port incisions and a 3-cm utility thoracotomy or a $\mathrm{CO}_{2}$ insufflation-combined assistant port, inserting a robot stapler through a 12 -mm port (16). In our institution, we currently use linear ports placed in the eighth intercostal space, as recommended by Intuitive Surgical. The company also recommends placing an assist port two ribs lower; however, we place the assist port in the fourth or fifth intercostal space on the anterior axillary line because assistant surgeons can reasonably access all lobes, hilar structures, and mediastinal tissues using this method (Figure 1A). We affix an alnote-LAPSINGLE (Alfresa; Osaka, Japan) to the wound retractor in the assist window (Figure 1B) and insert the AirSeal access port (ConMed, Utica, USA) into the one of the ports to allow for $\mathrm{CO}_{2}$ insufflation (Figure $1 \mathrm{~B}$ ). $\mathrm{CO}_{2}$ is then insufflated into the thoracic cavity at $8 \mathrm{mmHg}$ using the AirSeal iFS Intelligent Flow System (ConMed, Utica, USA). We use the complete portal robotic lobectomy (CPRL) technique with $\mathrm{CO}_{2}$ insufflation, "CPRL-4", developed by Cerfolio et al. (17). The pressure from the $\mathrm{CO}_{2}$ insufflation causes the mediastinum to retract and reduces respiratory fluctuations in the mediastinum, which widens the working space of thoracic cavity and facilitates the surgical manipulations. Regarding the robotic instrumentation (right side setup), we use the tip-up grasper on robotic arm 1 to retract the lung and other structures to improve the surgical view. On robotic arm 2, we use the fenestrated bipolar forceps to grasp tissues softly and accurately. On arm 4, we use the long bipolar grasper, which is longer than the Maryland forceps and has a stronger grip force. It is easy to perform both sharp and blunt tissue dissection and encircle the vessels safely using this instrument. We sometimes use a vessel sealer to seal and cut thick tissues and the small branches of blood vessels. Along with the DVSS generation 4 (da Vinci $X$ and $\mathrm{Xi}$ ), we started to use da Vinci staplers, and the EndoWrist ${ }^{\circledR}$ Stapler and SureForm ${ }^{\mathrm{TM}}$ (Intuitive Surgical), to staple the vessels, bronchus, and lung parenchyma. Although opinions differ regarding port placement and $\mathrm{CO}_{2}$ insufflation, including in Japan, we expect that more preferred and ideal robotic surgical procedures will be pursued and shared by thoracic surgeons.

\section{Comparison of VATS with RATS}

Although many studies of the perioperative outcomes and long-term prognosis following VATS have been published in Japan $(18,19)$, no studies comparing the surgical results of VATS with RATS are available, mainly because of its shorter history, in Japan. However, large comparative studies of long-term survival between VATS and RATS were recently performed at major institutions in the USA. Yang et al. compared long-term outcomes among 
RATS, VATS, and open approaches using propensityscore matching (20). The authors reported that 5-year overall survival after RATS, VATS, and open were $77.6 \%$, $73.5 \%$, and $77.9 \%$, respectively, and concluded that RATS resulted in similar long-term survival to VATS and thoracotomy. More recently, Huang et al. analyzed the long-term outcomes of anatomical lung resection by RATS or VATS (21). The authors did not perform propensityscore matching; however, there was no significant difference in the demographic data for the RATS and VATS groups. Regarding survival outcomes, the authors reported that RATS had significantly better overall survival compared with VATS $(89.2 \%$ in RATS and $74.7 \%$ in VATS; $\mathrm{P}=0.0292$, log-rank test). The authors reported that the results were confounded by a lower percentage of patients with diabetes in the RATS group and that the overall survival remained similar in both groups after controlling for this confounder. We reviewed 299 patients with primary lung cancer who underwent RATS or VATS lobectomy and mediastinal lymph node dissection at our institution and compared postoperative survival in 98 patients (49 patients in each group) whose background factors were adjusted by propensity-score matching (unpublished data). The 5 -year recurrence-free survival rate was not significantly different between the groups (VATS $v s$. RATS: $76.9 \%$ vs. $91.2 \%$, respectively; $\mathrm{P}=0.21$ ), and we concluded that RATS had promising results equivalent to VATS for mediumto long-term survival. Regarding this point, the ROMAN study, which is an ongoing prospective multicenter randomized trial (NCT02804893) to compare RATS and VATS approaches for early-stage lung cancer, will provide important and definitive information in the future (22).

\section{Useful techniques in RATS for advanced lung cancer}

The use of articulated robotic instruments in the thoracic cavity allows for more elaborate and complicated movements, and anastomosis is one of the most useful and advantageous techniques among several specialties using robotic surgery. However, RATS anastomosis is not as common among thoracic surgeons because transection is a major procedure, and organ reconstructions are rarely performed in thoracic surgery. From Japan, Nakamura et al. reported their first experience with robotic bronchoplastic upper lobectomy for squamous cell carcinoma of the right hilum of the lung (23). The authors used deep and wide wedge resection and interrupted sutures, and reported accidentally cutting the suture several times while ligating, mainly because of the absence of a tactile sense and difficulty in tension control despite the fact that the robotic anastomotic procedure was performed smoothly. A recent study by Jiao et al. from China (24) concluded that robotic bronchial sleeve lobectomy anastomosis was both feasible and safe for carefully-selected patients. Although robotic bronchial anastomosis appears to be a highly-promising procedure for central lung cancer and locally-advanced lung cancer that requires the technique, the technique must be simplified through further technological modifications.

Another reported important advantage of RATS compared with other approaches is lymph node dissection. Three-dimensional visualization and robotic instruments with more freedom degrees of motion permit thoracic surgeons to perform lymph node dissection properly and completely (25). Some studies suggested that RATS was equivalent to VATS for lymph node dissection $(26,27)$, whereas Toker et al. stated that RATS yielded significantly more total lymph nodes and the number of dissected hilar lymph nodes compared with other approaches (28). One of the reasons why more hilar lymph nodes were dissected was that it is possible to perform accurate sharp dissection of the vascular sheath around the pulmonary vessels and lymph nodes in RATS, unlike blunt dissection in VATS. Veronesi et al. preformed a retrospective multicenter study of 223 patients with clinically-evident or occult N2 lung cancer who underwent RATS lung resection (25). The authors suggested that RATS decreased the recovery time and the time between surgery and adjuvant treatments, and provided a higher chance of patients receiving full doses of chemotherapeutics, especially patients with advanced-stage cancer. Although it remains controversial whether more dissected lymph nodes lead to prognostic improvement, RATS may provide for accurate staging and avoid the disadvantages of under-staging. In Veronesi's study, 34 (15.2\%) patients received neoadjuvant treatment for N2-stage III non-small cell lung cancer. All 34 patients underwent R0 resection, 5 (15\%) patients required conversion but none required conversion because of bleeding, and 4 (12\%) patients had grade III or IV postoperative complications, suggesting that RATS was also safe and effective in patients with stage III non-small cell lung cancer who underwent neoadjuvant therapy. In Japan, RATS has been established as an approach for earlystage lung cancer; however, we expect that RATS will be used for highly-selected locally-advanced lung cancer after preoperative treatment, in the future.

Here we discuss our experience of RATS in a patient 

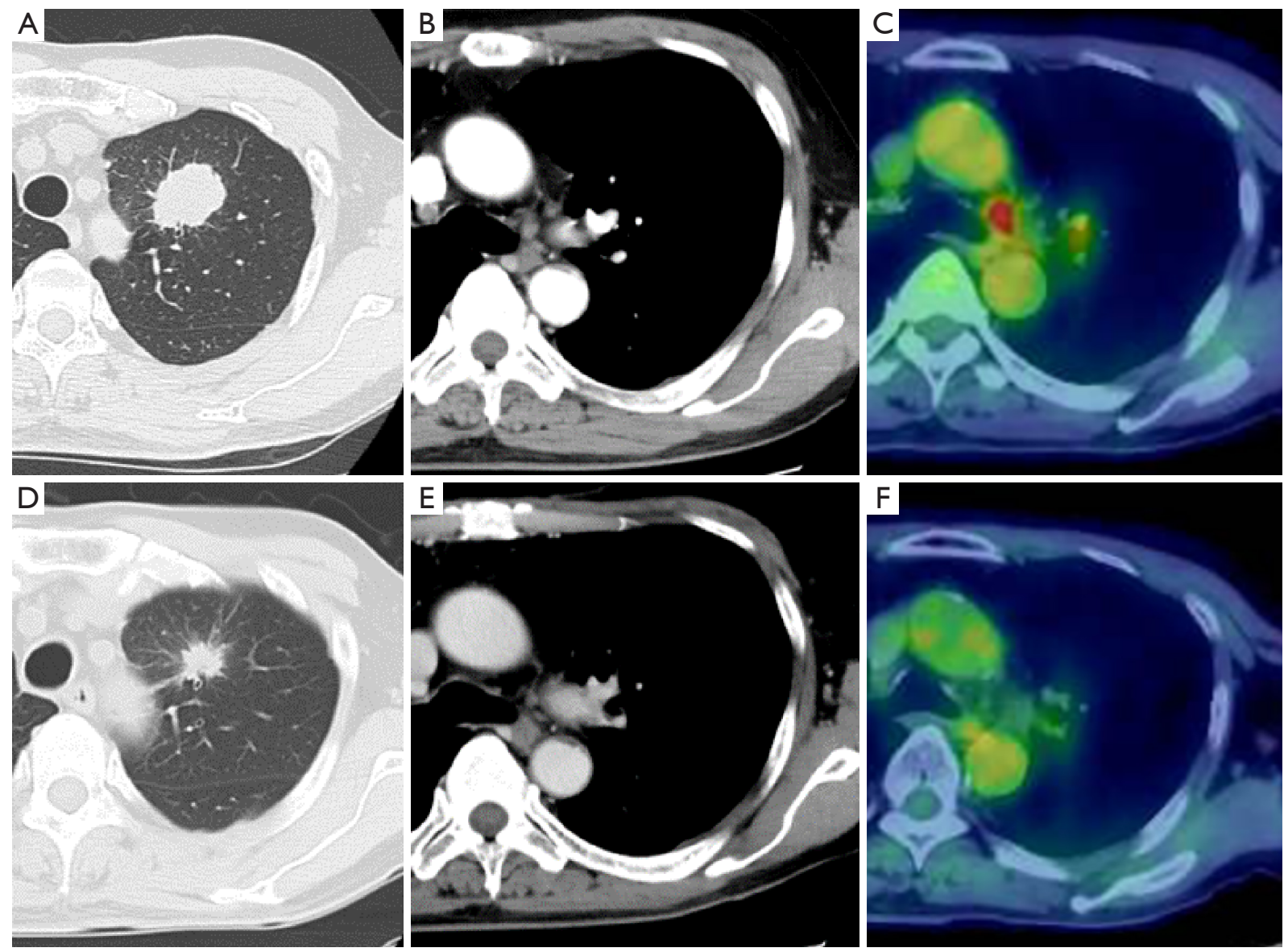

Figure 2 Imaging findings of the case. (A,B,C) A primary tumor in the left upper lobe and left upper mediastinal lymph node metastasis with fluorodeoxyglucose (FDG) uptake in positron-emission tomography/computed tomography (PET/CT). (D,E,F) After induction therapy with platinum-based doublet chemotherapy (cisplatin and docetaxel) and 50-Gy concurrent radiation therapy, both the primary tumor and the metastatic lymph nodes decreased in size, and no FDG uptake was seen on PET in the mediastinal lymph nodes.

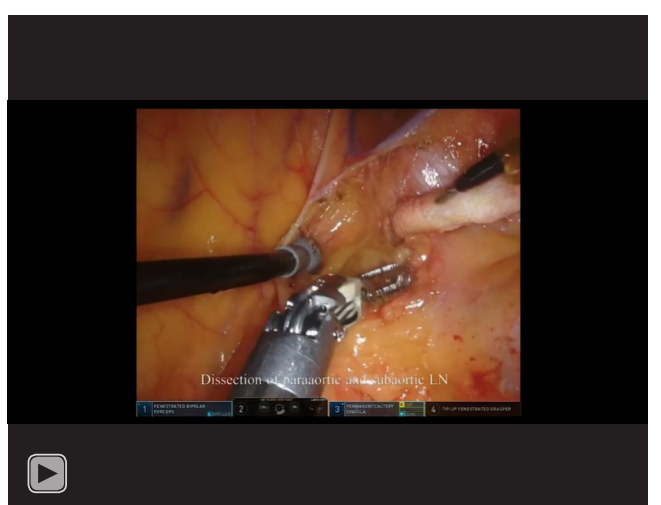

Video 1 Robot-assisted thoracoscopic surgery in left upper lobectomy with mediastinal lymph node dissection using the complete portal robotic lobectomy (CPRL) technique with carbon dioxide insufflation for primary lung cancer after induction therapy (29). with advanced lung cancer. The patient was a 66-year-old man and a former smoker, with a primary tumor in the left upper lobe with histologically-confirmed mediastinal lymph node metastasis. He initially received induction therapy with platinum-based doublet chemotherapy (cisplatin and docetaxel) and 50-Gy concurrent radiation therapy. Following treatment, both the primary tumor and metastatic lymph nodes decreased in size, and there was no longer any uptake of fluorodeoxyglucose (FDG) in mediastinal lymph nodes with positron-emission tomography (PET) (Figure 2). We then performed RATS left upper lobectomy with mediastinal lymph node dissection using the CPRL-4 technique (Video 1). First, we cut the mediastinal pleura along the left phrenic and vagal nerves, while exposing the left superior pulmonary vein, root of the left main pulmonary artery, and the aortic arch. 


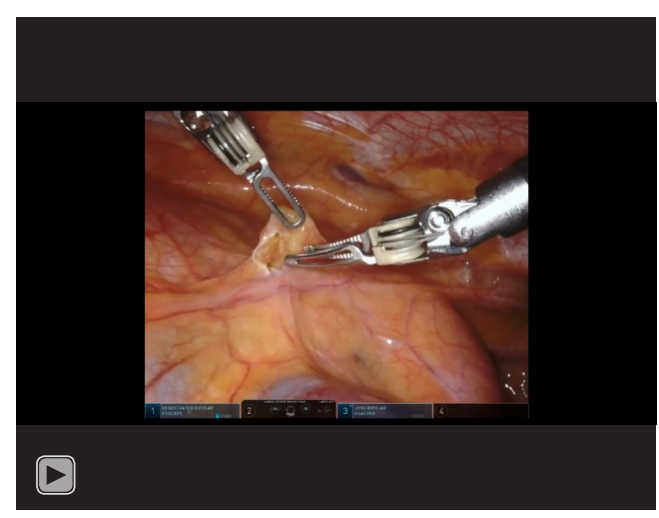

Video 2 Robot-assisted thoracoscopic surgery; thymectomy with a unilateral approach using three arms in the supine position for an anterior mediastinal tumor (30).

Next, we dissected the subaortic and paraaortic lymph nodes prior to lobectomy. The superior pulmonary vein and branches of the pulmonary arteries were encircled and cut with the da Vinci stapler after sharply dissecting the perivascular sheath. The orifice of the upper bronchus was then exposed and cut with the da Vinci stapler. After lobectomy, we opened the posterior mediastinal pleural along the vagal nerve, and bluntly dissected the peritracheal fat around the left side wall of the trachea, recurrent nerve, and aortopulmonary ligament. Lymph nodes around the left main bronchus were dissected from peripheral to central, and we sealed the root of the lymphatic tissue using a vessel sealer. Finally, we dissected the subcarinal lymph nodes anteriorly and posteriorly while exposing the inferior pulmonary vein, pericardium, and esophagus. All mediastinal fat tissue and lymph nodes were placed in a retrieval pouch. The total operation time and console time was 226 and 175 minutes, respectively. The patient had an uncomplicated postoperative course, and was discharged 7 days after surgery.

\section{RATS for mediastinal tumors}

Concurrently with lobectomy for pulmonary malignant tumors, RATS resection for mediastinal tumors was approved as an insurable procedure by the Japanese national health insurance system in April 2018. Regarding robotic surgical approaches for anterior mediastinal tumors, including thymic neoplastic diseases, there is still some debate regarding whether the lateral or subxiphoid approach is preferred. Many institutions in the USA,
European countries, and Japan use a unilateral approach with three arms in the supine position, and robotic operations performed with the lateral approach for anterior mediastinal tumors were reported in Japan (Video 2) (31-33). Meanwhile, Suda et al. emphasized the usefulness of the subxiphoid approach in RATS for anterior mediastinal tumors in a review (34). The authors stated that the subxiphoid approach makes it easier to identify the location of the bilateral phrenic nerves and offers good visualization in the neck because a camera is inserted through a subxiphoid incision placed on the midline, and a surgical field comparable to that in a median sternotomy can be achieved using this approach. Because both lateral and subxiphoid approaches have advantages and disadvantages, each surgeon and facility must select the approach with which they are familiar and wellexperienced. In both approaches, insufflating with $\mathrm{CO}_{2}$ may be useful because the thymus is located in the narrow anterior mediastinum.

In 2011, a group of surgeons experienced in minimally invasive thymic resections was assembled to review the definitions, terms, and procedures in the existing literature for minimally invasive thymoma resection. The group proposed a broad definition, namely, that a minimally invasive thymectomy includes "any approach" as long as no sternotomy (including partial sternotomy) or thoracotomy with rib spreading is involved and in which complete resection of the tumor is intended (35). The most important element in robotic thymic surgery is not the approach, but instead, surgical procedures that completely prevent tumor dissemination and local recurrence.

Posterior mediastinal tumors can also be good indications for robotic surgery. Kajiwara et al. reported three patients with mediastinal tumors located immediately adjacent to the vertebrae or aorta who underwent resection using the DVSS, and all robotic procedures were performed safely, smoothly, and extremely precisely. The authors concluded that crucial to the success of these operations was the appropriate placement and angle of the special da Vinci surgical ports in relation to the target and the patient's position, which varied according to the tumor location (36). Cerfolio et al. reviewed data for 75 patients with inferior or posterior mediastinal pathology who underwent robotic surgery and reported that although tumors located in the posterior and especially the inferior chest are sometimes difficult to approach robotically, specific techniques may help improve the operation (37). Further data for patients undergoing RATS for mediastinal 
tumors are needed to obtain definitive conclusions regarding the technical aspects.

\section{Risk management skills in RATS}

Operating-room crises such as massive bleeding or cardiac arrest are high-risk, stressful events that require rapid, coordinated, and time-critical care. In addition, these are situations in which the way the team cares for a patient makes the difference between life and death (38). Some previous reports demonstrated that the incidence of major vascular injury during robotic pulmonary resection was approximately $2 \%$, and that the most common catastrophic event was massive bleeding from the pulmonary arteries $(39,40)$. In Japan, more than two-thirds of vessel injuries during thoracoscopic surgeries involve branches of the pulmonary arteries and veins (41); therefore, it is mandatory for robotic thoracic surgeons to have the skills to cope with intraoperative catastrophic bleeding. Robotic surgery is not a solo surgery performed by a console surgeon alone. Because even a small mistake can be fatal, especially in minimally invasive thoracic surgery, including robotic surgery, anesthesiologists and paramedical staff, especially operating room nurses and medical engineers, work closely with each other peri- and intraoperatively, and must confirm important matters within the team. In addition, it is also important to perform emergency simulations regularly to prepare for severe intraoperative bleeding. Emergency simulations should be performed by the console surgeon and the assist surgeons with the anesthesiologists, operating room nurses, and medical engineers. It is necessary for them to check how the assist surgeon secures the space when converting from robotic surgery to thoracotomy and whether the rollout can be performed quickly. It is also important to create a role-specific assignment list for use in an emergency and to confirm each role during simulations. Although minimallyinvasive surgeries, including RATS, have gained popularity in the management of lung cancer and other chest diseases in Japan, as in other countries, these procedures are of little value unless they are performed adequately and safely. Thoracic surgeons should remember that these procedures have the possible risk of becoming catastrophic and "maximally-invasive" surgeries (42).

\section{Acknowledgments}

Funding: None.

\section{Footnote}

Provenance and Peer Review: This article was commissioned by the Guest Editor (Meinoshin Okumura) for the series "Dedicated to the 36th Annual Conference of Japanese Association for Chest Surgery" published in fournal of Visualized Surgery. The article has undergone external peer review.

Conflicts of Interest: All authors have completed the ICMJE uniform disclosure form (available at https://jovs. amegroups.com/article/view/10.21037/jovs.2019.12.08/ coif). The series "Dedicated to the 36th Annual Conference of Japanese Association for Chest Surgery" was commissioned by the editorial office without any funding or sponsorship. The authors have no other conflicts of interest to declare.

Ethical Statement: The authors are accountable for all aspects of the work in ensuring that questions related to the accuracy or integrity of any part of the work are appropriately investigated and resolved. All procedures performed in this study were in accordance with the Helsinki Declaration (as revised in 2013). The manuscript is waived from patient informed consent according to the ethics committee or institutional review board.

Open Access Statement: This is an Open Access article distributed in accordance with the Creative Commons Attribution-NonCommercial-NoDerivs 4.0 International License (CC BY-NC-ND 4.0), which permits the noncommercial replication and distribution of the article with the strict proviso that no changes or edits are made and the original work is properly cited (including links to both the formal publication through the relevant DOI and the license). See: https://creativecommons.org/licenses/by-nc-nd/4.0/.

\section{References}

1. Yoshino I, Hashizume M, Shimada M, et al. Thoracoscopic thymomectomy with the da Vinci computer-enhanced surgical system. J Thorac Cardiovasc Surg 2001;122:783-5.

2. Yoshino I, Hashizume M, Shimada M, et al. Video-assisted thoracoscopic extirpation of a posterior mediastinal mass using the da vinci computer enhanced surgical system. Ann Thorac Surg 2002;74:1235-7.

3. Morikawa T. Thoracoscopic surgery for lung cancer. Ann Thorac Cardiovasc Surg 2006;12:383-7. 
4. Shigemura N, Akashi A, Funaki S, et al. Long-term outcomes after a variety of video-assisted thoracoscopic lobectomy approaches for clinical stage IA lung cancer: a multi-institutional study. J Thorac Cardiovasc Surg 2006;132:507-12.

5. Sakuraba M, Miyamoto H, Oh S, et al. Video-assisted thoracoscopic lobectomy vs. conventional lobectomy via open thoracotomy in patients with clinical stage IA nonsmall cell lung carcinoma. Interact Cardiovasc Thorac Surg 2007;6:614-7.

6. Sawada S, Komori E, Yamashita M, et al. Comparison in prognosis after VATS lobectomy and open lobectomy for stage I lung cancer: retrospective analysis focused on a histological subgroup. Surg Endosc 2007;21:1607-11.

7. Murakawa T, Ichinose J, Hino H, et al. Long-term outcomes of open and video-assisted thoracoscopic lung lobectomy for the treatment of early stage non-small cell lung cancer are similar: a propensity-matched study. World J Surg 2015;39:1084-91.

8. Haruki T, Miwa K, Araki K, et al. Distribution and Prevalence of Locoregional Recurrence after VideoAssisted Thoracoscopic Surgery for Primary Lung Cancer. Thorac Cardiovasc Surg 2016;64:526-32.

9. Suda T, Sugimura H, Kitamura Y, et al. Robot-assisted major lung resection using the da Vinci Surgical System: The first case in Japan. Jpn J Chest Surg 2010;24:727-32.

10. Nakamura H, Suda T, Ikeda N, et al. Initial results of robot-assisted thoracoscopic surgery in Japan. Gen Thorac Cardiovasc Surg 2014;62:720-5.

11. Veronesi G, Galetta D, Maisonneuve P, et al. Four-arm robotic lobectomy for the treatment of early-stage lung cancer. J Thorac Cardiovasc Surg 2010;140:19-25.

12. Cerfolio RJ, Bryant AS, Minnich DJ. Starting a robotic program in general thoracic surgery: why, how, and lessons learned. Ann Thorac Surg 2011;91:1729-36; discussion 1736-7.

13. Dylewski MR, Ohaeto AC, Pereira JF. Pulmonary resection using a total endoscopic robotic video-assisted approach. Semin Thorac Cardiovasc Surg 2011;23:36-42.

14. Suda T. Transition from video-assisted thoracic surgery to robotic pulmonary surgery. J Vis Surg 2017;3:55.

15. Taniguchi Y, Nakamura H, Miwa K, et al. Initial Results of Robotic Surgery for Primary Lung Cancer: Feasibility, Safety and Learning Curve. Yonago Acta Med 2017;60:162-6.

16. Kanzaki M. Current status of robot-assisted thoracoscopic surgery for lung cancer. Surg Today 2019;49:795-802.

17. Cerfolio RJ, Bryant AS, Skylizard L, et al. Initial consecutive experience of completely portal robotic pulmonary resection with 4 arms. J Thorac Cardiovasc Surg 2011;142:740-6.

18. Nakao M, Ichinose J, Matsuura Y, et al. Long-term oncological outcome after thoracoscopic lobectomy for non-small cell lung cancer patients. J Thorac Dis 2019;11:3112-21.

19. Oda R, Okuda K, Osaga S, et al. Long-term outcomes of video-assisted thoracoscopic surgery lobectomy vs. thoracotomy lobectomy for stage IA non-small cell lung cancer. Surg Today 2019;49:369-77.

20. Yang HX, Woo KM, Sima CS, et al. Long-term Survival Based on the Surgical Approach to Lobectomy For Clinical Stage I Nonsmall Cell Lung Cancer: Comparison of Robotic, Video-assisted Thoracic Surgery, and Thoracotomy Lobectomy. Ann Surg 2017;265:431-7.

21. Huang L, Shen Y, Onaitis M. Comparative study of anatomic lung resection by robotic vs. video-assisted thoracoscopic surgery. J Thorac Dis 2019;11:1243-50.

22. Veronesi G, Cerfolio R, Cingolani R, et al. Report on First International Workshop on Robotic Surgery in Thoracic Oncology. Front Oncol 2016;6:214.

23. Nakamura H, Taniguchi Y, Miwa K, et al. A successful case of robotic bronchoplastic lobectomy for lung cancer. Ann Thorac Cardiovasc Surg 2013;19:478-80.

24. Jiao W, Zhao Y, Qiu T, et al. Robotic Bronchial Sleeve Lobectomy for Central Lung Tumors: Technique and Outcome. Ann Thorac Surg 2019;108:211-8.

25. Veronesi G, Park B, Cerfolio R, et al. Robotic resection of Stage III lung cancer: an international retrospective study. Eur J Cardiothorac Surg 2018;54:912-9.

26. Louie BE, Farivar AS, Aye RW, et al. Early experience with robotic lung resection results in similar operative outcomes and morbidity when compared with matched video-assisted thoracoscopic surgery cases. Ann Thorac Surg 2012;93:1598-604; discussion 1604-5.

27. Lee BE, Korst RJ, Kletsman E, et al. Transitioning from video-assisted thoracic surgical lobectomy to robotics for lung cancer: Are there outcomes advantages? J Thorac Cardiovasc Surg 2014;147:724-9.

28. Toker A, Ozyurtkan MO, Demirhan O, et al. Lymph Node Dissection in Surgery for Lung Cancer: Comparison of Open vs. Video-Assisted vs. Robotic-Assisted Approaches. Ann Thorac Cardiovasc Surg 2016;22:284-90.

29. Haruki T, Takagi Y, Kubouchi Y, et al. Robot-assisted thoracoscopic surgery in left upper lobectomy with mediastinal lymph node dissection using the complete portal robotic lobectomy (CPRL) technique with carbon 
dioxide insufflation for primary lung cancer after induction therapy. Asvide 2020;7:070. Available online: http://www. asvide.com/watch/33109

30. Haruki T, Takagi Y, Kubouchi Y, et al. Robot-assisted thoracoscopic surgery; thymectomy with a unilateral approach using three arms in the supine position for an anterior mediastinal tumor. Asvide 2020;7:071. Available online: http://www.asvide.com/watch/33110

31. Kajiwara N, Taira M, Yoshida K, et al. Early experience using the da Vinci Surgical System for the treatment of mediastinal tumors. Gen Thorac Cardiovasc Surg 2011;59:693-8.

32. Kajiwara N, Kakihana M, Kawate N, et al. Appropriate set-up of the da Vinci Surgical System in relation to the location of anterior and middle mediastinal tumors. Interact Cardiovasc Thorac Surg 2011;12:112-6.

33. Nakamura H, Taniguchi Y, Fujioka S, et al. First experience of robotic extended thymectomy in Japan for myasthenia gravis with thymoma. Gen Thorac Cardiovasc Surg 2012;60:183-7.

34. Suda T. Robotic subxiphoid thymectomy. J Vis Surg 2016;2:118.

35. Toker A, Sonett J, Zielinski M, et al. Standard terms, definitions, and policies for minimally invasive resection of thymoma. J Thorac Oncol 2011;6:S1739-42.

doi: 10.21037 /jovs.2019.12.08

Cite this article as: Haruki T, Takagi Y, Kubouchi Y, Kidokoro Y, Nakanishi A, Taniguchi Y, Nakamura H. Current status of robot-assisted thoracoscopic surgery in Japan. J Vis Surg 2020;6:29.
36. Kajiwara N, Kakihana M, Usuda J, et al. Extended indications for robotic surgery for posterior mediastinal tumors. Asian Cardiovasc Thorac Ann 2012;20:308-13.

37. Cerfolio RJ, Bryant AS, Minnich DJ. Operative techniques in robotic thoracic surgery for inferior or posterior mediastinal pathology. J Thorac Cardiovasc Surg 2012;143:1138-43.

38. Arriaga AF, Bader AM, Wong JM, et al. Simulationbased trial of surgical-crisis checklists. $\mathrm{N}$ Engl J Med 2013;368:246-53.

39. Cerfolio RJ, Bess KM, Wei B, et al. Incidence, Results, and Our Current Intraoperative Technique to Control Major Vascular Injuries During Minimally Invasive Robotic Thoracic Surgery. Ann Thorac Surg 2016;102:394-9.

40. Cao C, Cerfolio RJ, Louie BE, et al. Incidence, Management, and Outcomes of Intraoperative Catastrophes During Robotic Pulmonary Resection. Ann Thorac Surg 2019;108:1498-504.

41. Japan Society for Endoscopic Surgery. 12th Nationwide Survey of Endoscopic Surgery in Japan (in Japanese). J Jpn Soc Endosc Surg 2014;19:569-82.

42. Haruki T, Nakamura H. Response to intraoperative bleeding during video-assisted thoracoscopic surgery. J Vis Surg 2018;4:232. 